\title{
Electricity consumption management in a competitive electricity market
}

\author{
V. Oboskalov \& T. Panikovskaya \\ Ural Federal University named after the first President of Russia \\ B. N. Yeltsin, Russia
}

\begin{abstract}
A power system is characterized by the nonuniform nature of electric energy consumption. In the competitive electricity market, the electric energy cost is the spot one, which is determined for every fixed time interval and can significantly differ with the hours of maximum and minimum loads, on business and nonbusiness days, etc. The consumer is primarily interested in reducing the energy consumption during the peak load hours and shifting it onto the off load periods, which results in load leveling and allows for energy saving.

To solve the problem of improving the energy consumption reliability and safety, maintaining the power system stable operation as well as optimizing the electric energy cost, the following solutions can be proposed:

- limiting the energy consumption during the peak load periods and shifting it onto the off load periods;

- making long-term bilateral contracts on energy and power purchasing;

- locating the low capacity sources (distributed generation) near the consumers;

- introducing energy saving technologies into goods and services production.
\end{abstract}

The paper presents the analysis of the effect of energy consumption limitations on the consumer's profit in the short-term perspective. The loss function for consumers can be expressed mathematically in different ways. The criterion expressions for the energy consumption are defined provided the loss of profit is minimum. The consumer sector is shown to have to be of a greater regulating power in the electricity spot market operation.

Keywords: demand side management, competitive electricity market, limitation of power consumption, peak hours, optimal value restrictions, specific damage, schedule load. 


\section{Introduction}

A power system is characterized by the nonuniform nature of electric energy consumption during the day, which affects the price change in the wholesale and retail electricity markets and power. In fact, the price schedule follows the shape of the load curve grid and leads to an increase in prices during peak hours from 1.2 to 1.5 times.

Optimal solution to reduce energy consumption and reduce loads in the hours passing the maximum or other times is the widespread introduction and development of demand management technology [1-4]. Managing power consumption can be carried out using the economic and/or administrative methods. Economic methods involve the voluntary participation of consumers in control programs load at peak consumption and make energy efficiency advantage or disadvantage depending on the conditions of operation of the power provided to the consumer and economic incentives [5].

GOST 19431-84 introduces the following definition of the consumer load controlled: "the consumer of electricity or heat mode which provides the ability to limit power consumption or heat consumption in peak hours to equalize the load curve power system or power plant and increasing the load in hours minimum".

Consumers have the ability to regulate their load can:

- optimize daily load curve;

- voluntarily reduce or remove the peaks of consumption in response to high peak prices;

- participate in market power in order to reduce costs associated with the payment of the actual maximum power;

- impact on reducing power losses during transmission and distribution of electricity

- impose additional load (demand management use technology) to reduce market prices for electricity and additional benefits when buying electricity;

- while reducing peak demand on a market basis cede power liberated new customers interested in technological connection;

- provide services on a normalized primary frequency regulation, voltage level control, load management and consumption in critical and normal regimes in electric system.

Any restrictions or disconnecting the load give rise to damages for consumers. The magnitude of the damage depends on the following parameters [6, 7]: the degree of surprise restrictions consumer depth limit (off), duration, scope of the consumer, time of the restrictions. Limitations electricity consumer can be classified into:

- emergency with or without notice;

- targets determined by external factors, either related to a voluntary decision of the consumer.

Damage from the unplanned (emergency) blackouts or restrictions substantially higher than the planned [8], with the amount of damage depends on the depth 
and duration of the effect of surprise restrictions/outages, therefore the unit price will be different restrictions on the day-ahead market, balancing market, as well as during the automatic power system.

Currently in Russia there are no recommended regulators unified normative values for specific damages from outages consumers. Based on the international experience, the damage from sudden disconnections in problems of perspective development of EPS in the range of $2.5-4 \$ / \mathrm{kWh}$ and strongly depend on the type, power outages, as well as the activity of the enterprise or organization.

In order to achieve positive financial results some consumers the wholesale electricity market and power Russia today adjust their consumption, reducing the load during the hours of maximum prices, however, the effect of individual adjustment of individual subjects is not sufficient for a significant reduction in overall peak prices in the wholesale electricity market power [9].

On the day-ahead market customers (except guaranteeing suppliers) are allowed to submit three-stage downstream applications, but most consumers operate on the passive principle, feeding spot price acceptance of the application. As a result, the market is fully determined by the price bids of generators and one-sided. To stimulate consumers to active behavior in Russia is working to create economic mechanisms regulating ability to attract more consumers to reduce consumption during periods of high prices. This fee for reducing the burden to the consumer should be commensurate with the actual or anticipated damage that he is the introduction of restrictions.

Below we consider the problem of optimal configuration changes load schedule in order to reduce consumer payments for electricity at a predetermined interval on a daily single marginal price. A mathematical model for the economic evaluation of the load limiting function based on the use of specific damage from power limitations and power consumption.

\section{Mathematical models to assess damage}

\subsection{Defining a function of electrical energy prices}

Time-varying price of electricity at the wholesale or retail markets has a probabilistic nature determined by the stochastic nature of electricity, and cannot be exactly predicted in advance $[7,10]$. Without great error can assume that for each time $t$ price, as the load is described by the normal distribution with mean and variance $\bar{p}_{t}, \sigma_{p t}^{2}$. At the same time, in some cases for the analysis of economic laws is admissible deterministic formulation of the problem, where the daily price schedule presents a chronological series $\left\{p_{t}, t=1, \ldots, T\right\}$.

\subsection{Damage assessment modeling different functional dependencies}

Change in the price of electricity depends on the demand and leads to a wide range of prices, from the minimum - at night, to the maximum peak loads $[6,10$, 11]. Increasing prices increase enterprise losses related to payments for electricity and pay for power. Economic losses from high electricity prices can 
be reduced by limiting the power consumption by the amount $\Delta L$. To solve any problems of the market related to the optimization of payments for electricity and capacity as one of the key performance indicators used by the damage from the load limiting $y(\Delta L)=y(\Delta L) \Delta L, y(\Delta L)$ - specific damage of load limiting.

Specific damage depending on the relative depth limit load $\varepsilon=\Delta L / L_{p l}=$ $1-L / L_{p l}$ may be represented by different functional dependences:

- linear function $y(\varepsilon)=\alpha+\beta \varepsilon$;

- power function $y(\varepsilon)=\alpha+\beta \varepsilon^{\gamma}$;

- piecewise linear function

$$
y(\varepsilon)=\alpha+\beta_{1}\left(\varepsilon-\varepsilon_{1}\right)+\sum_{\varepsilon_{i}<\varepsilon}\left(\beta_{i}\left(\varepsilon_{i}-\varepsilon_{i-1}\right)\right), \varepsilon_{k}=\max \left\{\varepsilon_{i}<\varepsilon\right\} .
$$

The objective function representing the cost savings from limiting the power and electric energy on the current interval $\Delta t=1$ hour $(e(x)=\Delta L \Delta t)$ is described by

$$
e(x)=p x-y(\varepsilon) x=(p-y(\varepsilon)) x \rightarrow \max ,
$$

$p$ - price of electrical energy in the considered hour interval; $x$ - value of the load limiting: $x_{t}=\Delta L_{t}=\varepsilon_{t} L_{t} ; x=\Delta L=\varepsilon L ; \varepsilon=x / L-$ load limit in relative units.

The maximum value of the function $e(x)$ is determined from the condition

$$
\frac{d e(x)}{d x}=p-y(\varepsilon)-y^{\prime}(\varepsilon) \varepsilon=0 .
$$

Solution of this equation describes the optimum depth limitations and the maximum effect of $e(\hat{\varepsilon})$, obtained at the current market price for electricity.

Typically, the function of specific damage $y(\varepsilon)$ is non-decreasing $y^{\prime}(\varepsilon) \geq 0$, and the study of qualitative regularities further assumption is that it is also convex and $y^{\prime \prime}(\varepsilon) \geq 0$. In this case, the second derivative of the effectiveness (2) has the form

$$
\frac{d^{2} e(x)}{d x^{2}}=p-y(\varepsilon)-y^{\prime}(\varepsilon) \varepsilon=-\frac{\left[\frac{2 y^{\prime}(\varepsilon)}{L}+y^{\prime \prime}(\varepsilon)\right]}{L} \leq 0 .
$$

Hence, we consider the extreme point is a function of the maximum efficiency.

\subsubsection{Linear function of specific damage}

Linear characteristic of specific damage at expression (2) takes the form

$$
p-\alpha-2 \beta \varepsilon=\Delta \alpha-2 \beta \varepsilon=0 .
$$

Satisfying this relation optimal (by maximum efficiency) limit value $\hat{\varepsilon}$ is determined from value for money, constant and variable components of specific damage

$$
\widehat{\varepsilon}=\Delta \alpha /(2 \beta)
$$

Expression for the current load $L$ will have the form 


$$
L=\left\{\begin{array}{cc}
L, & p<\alpha ; \\
(1-\varepsilon) L, & \alpha \leq p \leq \alpha+2 \beta ; \\
0, & p>\alpha+2 \beta .
\end{array}\right.
$$

If the price $p$ less than specific load limiting damage from $\alpha$, then the restriction is inappropriate. If the price $p$ is greater than the proportion of the load limiting damage $\alpha+2 \beta$, the fee for electricity is so great that it is advisable to completely stop the main production. In the case of the intermediate load limit logical units in an amount relative to the $\varepsilon$ depends on the ratio given in expression (4).

According to (4), the function of the current economic benefit at an optimum time interval limitation of power consumption may be represented by three components, depending on the value for the specific component damage

$$
e(x)=(\Delta \alpha-\beta \widehat{\varepsilon}) \hat{\varepsilon} L=\beta \widehat{\varepsilon}^{2} L=\left\{\begin{array}{c}
L \frac{\Delta \alpha^{2}}{4 \beta}, p<\alpha \\
(1-\widehat{\varepsilon}) L \frac{\Delta \alpha^{2}}{4 \beta}, \alpha \leq p \leq \alpha+2 \beta \\
0, p>\alpha+2 \beta .
\end{array}\right.
$$

\subsubsection{Power function of specific damage}

When using power function descriptions of specific damage expression (2) takes the form

$$
\begin{gathered}
\frac{d e(x)}{d x}=\Delta \alpha-(1+\gamma) \beta \varepsilon^{\gamma}=0, \\
\hat{\varepsilon}=\left(\frac{\Delta \alpha}{(1+\gamma) \beta}\right)^{\frac{1}{\gamma}} .
\end{gathered}
$$

We introduce an additional substitution $\mu=1+1 / \gamma$

$$
e(x)=\left(\Delta \alpha-\beta \hat{\varepsilon}^{\gamma}\right) \hat{\varepsilon} L\left\{\begin{array}{c}
L \frac{\gamma \cdot \Delta \alpha^{\mu}}{\beta^{\gamma}(1+\gamma)^{\mu}}, p<\alpha \\
(1-\widehat{\varepsilon}) L \frac{\gamma \cdot \Delta \alpha^{\mu}}{\beta^{\gamma}(1+\gamma)^{\mu}}, \alpha \leq p \leq \alpha+(1+\gamma) \beta \\
0, p>\alpha+(1+\gamma) \beta .
\end{array}\right.
$$

It should be noted that when $\gamma=1$ expression (5) and (8) are identical.

Similar to the previous case, the determining optimal interval values limitations in describing specific damage piecewise linear function, which, depending on the specifics of algorithmic can be expressed near submissions. 


\subsubsection{Piecewise linear function of specific damage}

A. Representation of the solution of piecewise linear function

$$
y(\varepsilon)=\alpha_{k}+\beta_{k}\left(\varepsilon-\varepsilon_{k-1}\right), \varepsilon_{k-1} \leq \varepsilon<\varepsilon_{k}, k=1, \ldots, K .
$$

Taking into account the relation (2), the optimal value $\hat{\varepsilon}$ is algorithmically determined by the sequential viewing intervals linearity.

If $\widehat{\varepsilon}_{k}=\left(p-\alpha_{k}\right) /\left(2 \beta_{k}\right)>\varepsilon_{k+1}$, it should go to the next interval linearity. By analogy with (4) is checked interval boundaries determine the function of specific damage.

Algorithmically equivalent to (interval standardized view) representation of the result is obtained in the form of specific damage

$$
y(\varepsilon)=\alpha+\beta_{k}\left(\varepsilon-\varepsilon_{k-1}\right)+\sum_{\varepsilon_{i}<\varepsilon}\left(\beta_{i}\left(\varepsilon_{i}-\varepsilon_{i-1}\right)\right), \varepsilon_{k}=\max \left\{\varepsilon_{i}<\varepsilon\right\} .
$$

B. Representation of the solution in a multi-dimensional (linear in each interval) vector

$$
y(\varepsilon)=\alpha_{0}+\sum_{i=1}^{K}\left(\beta_{i}\left(x_{i}-\varepsilon_{i-1}\right)\right), \varepsilon=\sum_{i=1}^{K}\left(x_{i}-\varepsilon_{i-1}\right),
$$

provided that

$$
y\left(\varepsilon_{i}\right)=y\left(\varepsilon_{i-1}\right)+\beta_{i}\left(\varepsilon_{i}-\varepsilon_{i-1}\right) ; \varepsilon_{i-1} \leq x_{i}<\varepsilon_{i}-\varepsilon_{i} .
$$

As a result, an optimization problem is solved

$$
\max \{e(x)=p \varepsilon L-y(\varepsilon) \varepsilon L\}, \varepsilon=\sum_{i=1}^{K}\left(x_{i}-\varepsilon_{i-1}\right), \varepsilon_{i-1} \leq x_{i}<\varepsilon_{i}-\varepsilon_{i} .
$$

It is easy to see that the second approach is mathematically more complicated and more efficient. However, if the task of selecting the optimal loads limiting individual consumer is a component of some external optimization problem (for example, the choice of control actions in order to optimize energy consumption in some regions), it is possible that this approach will be in demand.

\subsection{Limitation of power consumption as an integral function}

The optimum load control while reducing power consumption for day interval defined quite simply: as the sum of the hourly restrictions. More interesting is the problem of re-load day interval with constant power consumption.

\subsubsection{Optimal load management without changing consumption day interval}

Mathematically, this problem can be formulated as follows. Find the optimal distribution of load restrictions $x_{t}, t=1, \ldots, 24$, resulting in maximum savings associated with the redistribution of power consumption for the day interval. In the analysis of specific damage, the representation of $y(\varepsilon)$ as a linear function

$$
E(X)=\max \sum_{t=1}^{24}\left(p_{t} x_{t}-y\left(\varepsilon_{t}\right) x_{t}\right)=\max \sum_{t=1}^{24}\left(p_{t}-y\left(\varepsilon_{t}\right)\right) x_{t}
$$

provided 


$$
\sum_{t=1}^{24} x_{t}=0, x_{t} \leq L_{t}, \forall t
$$

Solution of the problem can be found by the method of Lagrange. Condition presented in the form of restriction in equation (13) may be taken into account by introducing an additional vector of Lagrange multipliers. In this complementary slackness condition implies that if (13) is satisfied (restriction is not active), then the corresponding undetermined Lagrange multiplier is zero, and if active, $x_{t}=L_{t}$ and this variable can be deduced from the number of the variables. Hence, the optimization problem can be seen in the simplified formulation without (13).

The Lagrange function has the form

$$
L a g=\sum_{t=1}^{24}\left(p_{t}-y\left(\varepsilon_{t}\right)\right) x_{t}-\lambda \sum_{t=1}^{24} x_{t}
$$

Its differentiation and equating to zero the derivative leads to criteria condition

$$
\begin{gathered}
\nabla \operatorname{Lag}_{x_{t}}=p_{t}-\alpha-2 \beta \varepsilon_{t}-\lambda=0, t=1, \ldots, 24 ; \\
\nabla \operatorname{Lag}_{\lambda}=\sum_{t=1}^{24} \varepsilon_{t}=0 .
\end{gathered}
$$

Thus, the assumptions made optimal limit electricity proportional to the deviation in the market price for electricity from some settlement price difference and the constant component specific damage $\varepsilon_{t}=\left(p_{t}-\alpha-\lambda\right) / 2 \beta$.

Condition (15) allows us to determine $\lambda$. Undetermined Lagrange multiplier equal to the difference of the average price and the constant component specific damage - the coefficient $\alpha$, then the optimal limit on the hourly interval will take the following expression

$$
\lambda=\frac{1}{24} \sum_{t=1}^{24}\left(p_{t}-\alpha\right)=\bar{p}-\alpha,
$$

$\bar{p}-$ the average price on day interval.

$$
\varepsilon_{t}=\frac{p_{t}-\bar{p}}{2 \beta}, \sum_{t=1}^{24} \varepsilon_{t}=0, \varepsilon_{t} \leq L_{t}, \forall t .
$$

At a price higher than the average power consumption limitation advisable due to its increase in the hours when electricity costs are lower than average.

Total savings for constant power consumption for continuous time interval can be defined as follows 


$$
\begin{aligned}
E(X)=\int_{0}^{T} p(t) \varepsilon(t) d t & =\frac{1}{2 \beta} \int_{0}^{T} p(t)(p(t)-\bar{p}) d t= \\
= & \frac{\bar{p}^{2}}{2 \beta}\left(\sum_{t=0}^{T} \frac{p^{2}(t)}{\bar{p}^{2}} d t-T\right)=\frac{\bar{p}^{2} T}{2 \beta}\left(k_{f}^{2}-1\right)
\end{aligned}
$$

where $k_{f}$ - form factor of the daily price schedule.

The resulting relation is valid for any price, including the price and subject to surcharges and discounts coefficient.

\subsubsection{Optimal load management savings and consumption unchanged at day interval}

For the new price schedule estimated savings will be (with regard to the expression (18)

$$
E(X)^{*}=\frac{\tilde{p}^{2} T}{2 \beta}\left(\tilde{k}_{f}^{2}-1\right),
$$

$\tilde{p}, \tilde{k}_{f}$ - new average price and form factor

Consequently, it is necessary to calculate the average price of a new form factor and for the corrected schedule. The new average price can be calculated as given allowances and discounts $\theta$ [12], and without.

Assuming a ratio of premiums/discounts new average price will be equal

$$
\tilde{p}=\frac{1}{T} \int_{t=o}^{T} p^{*}(t) d t=p_{a w}+\left(\bar{p}-p_{a w}\right) \theta,
$$

$p_{a w}$ - average weighted price.

Root mean square (RMS) value of the square of the new prices for the corrected load curve assuming a ratio of surcharges and discounts $\theta$

$$
\tilde{p}_{r m s}^{2}=p_{a w}(1-\theta)(\tilde{p}+\theta \bar{p})+\theta^{2} p_{r m s}^{2} .
$$

New form factor

$$
\tilde{k}_{f}^{2}=\frac{\tilde{p}_{r m s}^{2}}{\tilde{p}^{2}}=1+\theta^{2} \frac{\bar{p}^{2}}{\tilde{p}^{2}}\left(k_{f}^{2}-1\right) .
$$

The proposed method of determining the values of load shedding allows the operator to market effectively manage processes optimizing the consumption of electric energy through market mechanisms. Overall reduction in energy consumption by redistributing the load curve and the introduction of allowances, discounts to the price - the most effective demand management strategy. 


\section{Test calculations}

\subsection{Example 1}

As a test example restrictions on the power of the load is considered day interval specified price schedule and the function of specific damage, provided the linear and the degree of dependence. The function of specific damage in relative terms has the form $y(\varepsilon)=1+0,4 \varepsilon$. The coefficient $\alpha$ in named units equals $950 \mathrm{rub} / \mathrm{MWh}$. Table 1 shows the calculated optimum values of load restrictions, in Figures 1 and 2 the price chart, the source and the adjusted load schedules.

Table 1: Load restrictions for linear characteristics of specific damage.

\begin{tabular}{|c|c|c|c|c|c|c|c|c|}
\hline Hour & 1 & 2 & 3 & 4 & 5 & 6 & 7 & 8 \\
\hline$p, \frac{\mathrm{rub}}{\mathrm{MWh}}$ & 901 & 875 & 768 & 664 & 730 & 788 & 800 & 949 \\
\hline$L, \mathrm{MW}$ & 8.82 & 8.31 & 8.31 & 8.31 & 8.57 & 8.98 & 10.0 & 11.9 \\
\hline$\varepsilon, r . \mathrm{u}$. & 0.00 & 0.00 & 0.00 & 0.00 & 0.00 & 0.00 & 0.00 & 0.00 \\
\hline$\Delta L, \mathrm{MW}$ & 0.00 & 0.00 & 0.00 & 0.00 & 0.00 & 0.00 & 0.00 & 0.00 \\
\hline hour & 9 & 10 & 11 & 12 & 13 & 14 & 15 & 16 \\
\hline $\begin{array}{c}\text { rub } \\
\text { MWh }\end{array}$ & 987 & 1047 & 1066 & 1047 & 1060 & 1064 & 1056 & 1035 \\
\hline$L, \mathrm{MW}$ & 13.1 & 13.4 & 13.4 & 13.2 & 13.17 & 13.13 & 13.2 & 13.2 \\
\hline$\varepsilon, r . \mathrm{u}$. & 0.05 & 0.13 & 0.15 & 0.13 & 0.15 & 0.15 & 0.14 & 0.11 \\
\hline$\Delta L, \mathrm{MW}$ & 0.64 & 1.71 & 2.05 & 1.69 & 1.91 & 1.97 & 1.84 & 1.49 \\
\hline hour & 17 & 18 & 19 & 20 & 21 & 22 & 23 & 24 \\
\hline$p, \frac{\mathrm{rub}}{\mathrm{MWh}}$ & 1028 & 986 & 969 & 945 & 963 & 954 & 955 & 912 \\
\hline$L, \mathrm{MW}$ & 13.2 & 13.36 & 13.5 & 13.6 & 13.9 & 14.2 & 12.0 & 10.1 \\
\hline$\varepsilon, r . \mathrm{u}$. & 0.10 & 0.05 & 0.03 & 0.00 & 0.02 & 0.01 & 0.01 & 0.00 \\
\hline$\Delta L, \mathrm{MW}$ & 1.91 & 1.97 & 1.84 & 1.49 & 1.36 & 0.63 & 0.34 & 0.00 \\
\hline
\end{tabular}

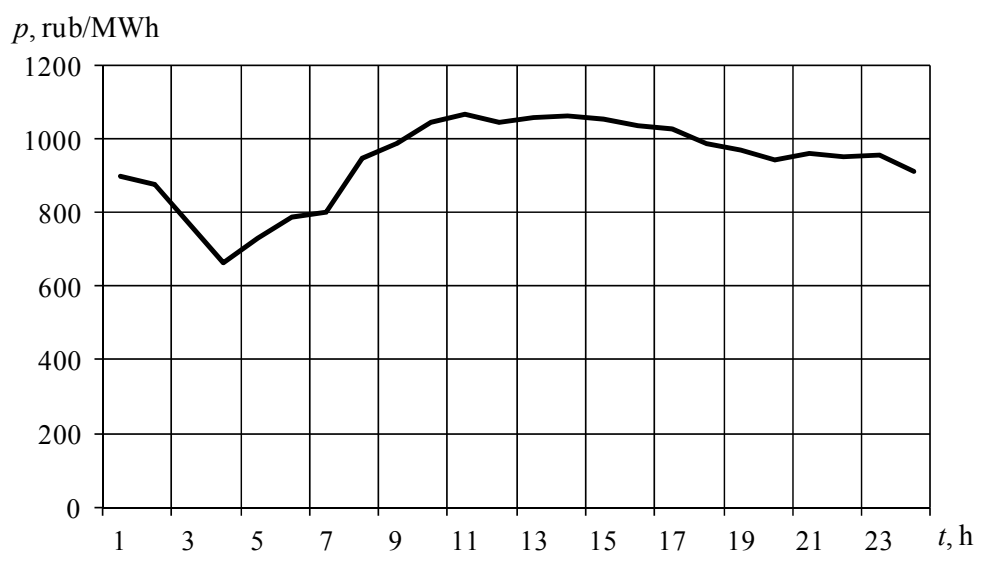

Figure 1: The price chart in the wholesale market of electric energy and power. 


\section{$L, \mathrm{MW}$}

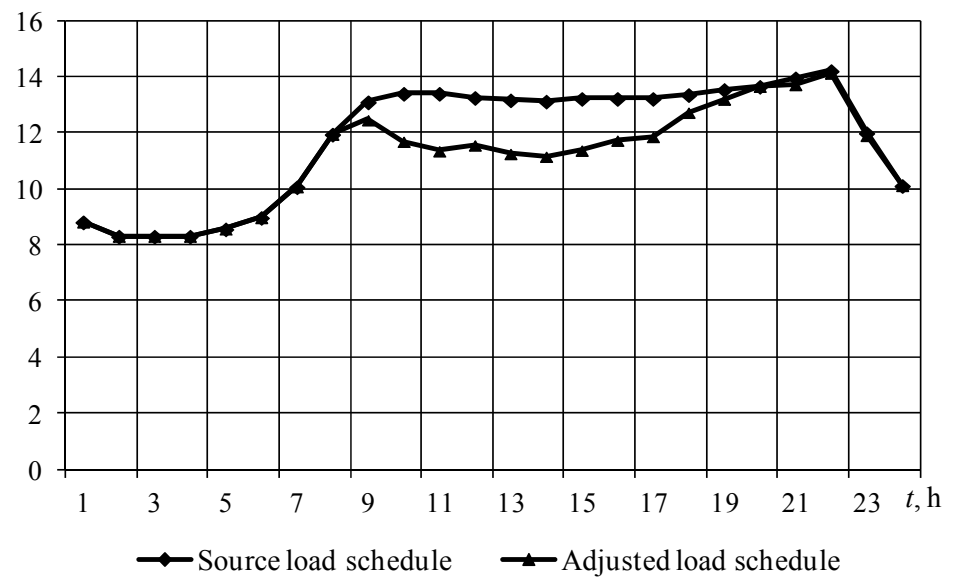

Figure 2: Source and adjusted graphics load.

Load limit depends on the price chart, the values of which are given in Table 1 and Figure 1. In the interval from 9 to 19 hours of electricity price exceeds the constant component of specific damage, so it is in this interval is advisable to limit power consumption. For the interval in question and the introduction of the calculated limit savings per day is $5.5 \%$. On the remaining interval calculation results show that the value of $p \leq \alpha$ limit load power is impractical.

\subsection{Example 2}

Example 1 was used to determine the optimal load transfer without changing the power consumption for the day interval. Main characteristics of the daily schedule are shown in Table 2.

Table 2: Characteristics of the daily price schedule.

\begin{tabular}{|c|c|c|}
\hline Maximum price, rub/MWh & $p_{\max }$ & 1066.1 \\
\hline Minimum price, rub/MWh & $p_{\min }$ & 664.8 \\
\hline Weighted average price for the load, rub/MWh & $p_{a w}$ & 956.9 \\
\hline Average price, rub/MWh & $\bar{p}$ & 946.5 \\
\hline Average load power, MW & $L_{a}$ & 11.8 \\
\hline RMS price, rub/MWh & $p_{r m s}$ & 961.3 \\
\hline Form factor & $k_{f}$ & 1.01 \\
\hline
\end{tabular}


Depending on the ratio of the constant depending on the ratio of the constant component of specific damage and the current market price of load transfer on the night hours is economically justified. Total savings for the given price schedule for the day is $1.49 \%$ of the total payment.

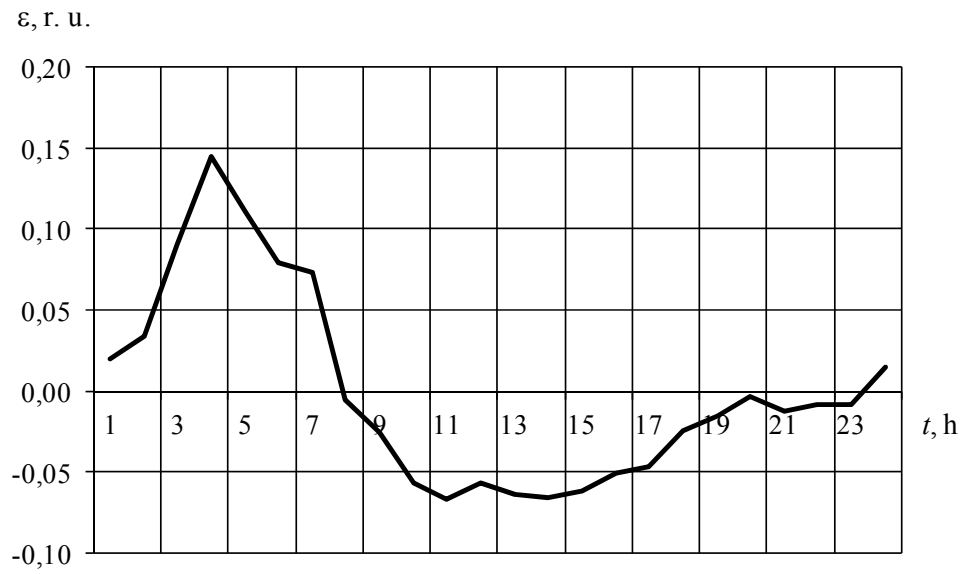

Figure 3: Optimal redistribution of the load curve.

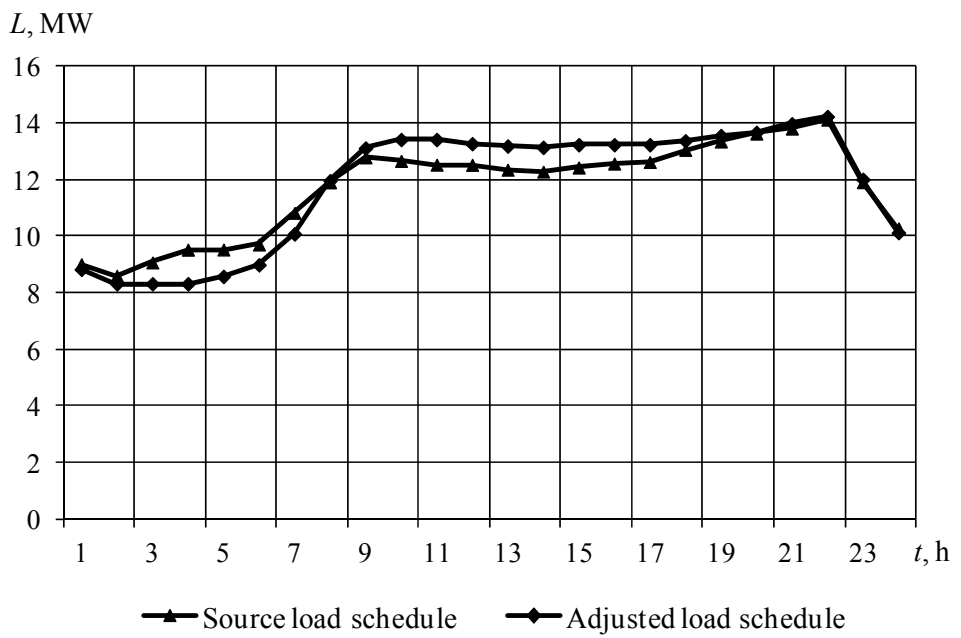

Figure 4: Redistribution of the load at a constant consumption per day. 


\section{Conclusion}

1. A wide range of changes in prices for electricity between the hours of minimum and maximum loads stimulates consumers to load redistribution time provided a possible change in the process of the enterprise over time.

2. Relevance of introducing energy management programs and energy efficiency depends on the economic interest of consumers in the voluntary participation of regulation of the load curve.

3. With increasing prices for electricity during peak hours replacing ineffective expensive generation load reduction on the part of the consumer can bring a positive effect on the overall market. Limiting the power to the consumer reduces its yield, but may be offset by a reduction in the cost of payments maximum power and energy) and, ultimately, lead to an increase in total profit.

4. Decision to limit the load during peak prices or transfer consumption periods of lower prices can and should make the consumer himself or through organizational and technical measures.

5. Consumers, reducing their power consumption, reduce the fee for the actual maximum power, are able to assign a market-based liberated power to other consumers, to improve their competitiveness.

6. Develop and implement programs that encourage consumers to reduce electricity demand during peak hours, will allow more fully utilize existing generating capacity, reduce uneven daily load curve, to get a system-wide energy-saving effect. Consumer sector should play a more active and regulatory role in the functioning of electricity and capacity markets.

\section{References}

[1] Demand Side Management, www.DemandSideManagement.com

[2] Implementing Agreement on Demand-Side Management Technologies and Programs 2009. International Energy Agency. Annual Report, January, 2010.

[3] Electricity Supply and Demand Side Management Options. Review Paper Final Circulation Draft, 24 March, 2000.

[4] Gillingham, K., Newell, R., Palmer, K., Retrospective Examination of Demand-Side Management Energy Efficiency Policies. Washington, 2004.

[5] Zgrurovets, O.V., Kostenko, G.P., Effective methods for managing the consumption of electrical energy. Problems zagalnoi Energy and Utilities. Kiev, 16, pp. 75-80, 2007.

[6] Aalami, H.A., Moghaddam, M.P., Yousefi G.R., Demand response modeling considering Inter-ruptible/Curtailable loads and capacity market programs. Applied Energy, 87, pp. 243-250, 2010.

[7] Stoft, S., Power System Economics. Designing Markets for Electricity. IEEE Press: Wiley - Interscience, 2002.

[8] Nepomnyaschy, V.A., Economic problems of increase of reliability of reliability of power supply. Tashkent: Fan, 1985. 
[9] Schweppe, F.C., Caramanis, M.C., Tabors, R.D., Bohn, R.E., Spot Pricing of Electricity. Kluwer Academic Publishers, 1988.

[10] Trufanov, V.V., Khanaev, V.V., Electric load management: opportunities and prospects. Electric Station, 2, pp. 2-6, 2011.

[11] Lamoureux, M.A., Palamarchuk, S.I., Voropai, N.I., Current Developments in Russian Power Industry Restructuring. Proc. of the Int. Conf. "Liberalization and Modernization of Power Systems: Congestion Management Problems”. Irkutsk: Energy Systems Institute, pp. 5-12, 2003.

[12] Oboskalov, V.P., Differentiated in time allowances and discounts on tariffs for electricity. Proc. of AN. Energetika, 6, pp. 54-62, 1998. 